\title{
Larvicidal Activity of Brusatol Isolated from Brucea javanica (L) Merr on Culex quinquefasciatus
}

\author{
*Dwi SUTININGSIH ${ }^{1}$, Nurjazuli NURJAZULI ${ }^{2}$, Djoko NUGROHO $^{3}$, Tri Baskoro \\ Tunggul SATOTO ${ }^{4}$
}

1. Department of Epidemiology and Tropical Disease, Faculty of Public Health, Diponegoro University, Semarang, Indonesia

2. Department of Environmental Health, Faculty of Public Health, Diponegoro University, Semarang, Indonesia

3. Department of Biostatistics, Faculty of Public Health, Diponegoro University, Semarang, Indonesia

4. Department of Parasitology, Faculty of Medicine, Gadjah Mada University, Yogyakarta, Indonesia

*Corresponding Author: Email: dwisuti98@gmail.com

(Received 10 Jan 2018; accepted 15 Apr 2018)

\begin{abstract}
Background: Vector control is still emphasized on the using of chemical insecticide which can cause death of non-target organisms, pollution and vector resistance. Therefore, natural insecticides/larvicides are an alternative to using chemical insecticides to control the mosquito vector.

Methods: Brusatol was isolated from the seeds of Makassar Fruit (Brucea javanica L. Merr). Culex quinquefasciatus larvae were divided into 3 groups, i.e. 6 testing groups and one negative and positive control group. In the negative control group, the larvae were treated with $100 \mathrm{ml}$ aquadest and positive control was treated with temephos $1 \mathrm{ppm}$. After 24 hours, dead larvae were calculated and the percentage of death was determined. The dead larvae were then examined for morphological changes using a light microscope.

Results: The higher of the concentration level of brusatol, the higher number of the death of $C \times$. quinquefasciatus larvae $(P<0.05)$. The value of brusatol Lethal Concentration 50 to larvae $C \times$. quinquefasciatus was $0.010 \pm 0.122$ and value of brusatol Lethal Concentration 90 to larvae $C \times$. quinquefasciatus was $0.654 \pm 0.081 \mathrm{ppm}$. The higher the concentration of brusatol, the morphological damage of $C x$. quinquefasciatus larvae was getting worse and widespread to cause damage to the digestive tract and cuticle.

Conclusion: Brusatol isolated from the seed of B. javanica (L) Merr have larvicidal activity to the Cx. quinquefasciatus which is potential to be natural larvicide.
\end{abstract}

Keywords: Larvicide; Brusatol; Brucea javanica (L) Merr; Culex quinquefasciatus

\section{Introduction}

The efforts of vector control in order to suppress the incidence of vector-borne disease to this day are still being attempted. The use of chemical insecticides and synthetic organism widely in vector control is endangering the environment and causing he resistance in vector (1). Therefore, it is necessary to find the alternative way to control the vector disease, that is using natural insecti- cides/larvicides that come from plants (2). Indonesia is rich of plants that have potential as natural insecticides/larvicides, and many of them have not yet proven their effectiveness scientifically (3).

Brucea javanica (L) Merr is known rich for quassinoid compounds, such as brusatol, bruceantine and bruceine (4). Several researches 
on quassinoids showed high potential as natural source of insecticides/larvicides. Quassinoids proven as compounds that responsible to the change of feeding behavior and growth regulation on insects (5). Quassin, simalikalactone $\mathrm{D}$, bruceantine, gluocarubinone and isobruceine A have been proven to be effective as antifeedant against Mexican bean beetles (Epilachna varivestis), cabbage caterpillar (Plutella xylostela) and south caterpillar (6). Brusatol isolated from B. javanica (L) Merr has insecticidal effect and antifeedant on larvae instar 3 of Spodoptera exigua (7). Brusatol can induce apoptosis on insect cell line IOZCASSpec-II and Sf21 characterized by DNA fragmentation, kaspace-3 activation and the release of cytochrome-c enzyme from mitochondria (7).

Larvicidal activities of brusatol on Aedes aegypti larvae with Lethal Concentration value of 50 and $90\left(\mathrm{LC}_{50}, \mathrm{LC}_{90}\right)$ are $0.669 \pm 0.106 \mathrm{ppm}$ and 8.331 $\pm 0.060 \mathrm{ppm}$ (8). The treatment with various brusatol concentration on $A$ e. aegypti larvae showed neurotoxic syndromes that were exitacy, convulsion, tremor and paralysis (8). Another quassinoid compound contained in B. javanica (L) Merr that is bruceine A also shown to prohibit the growth and development of Ae. aegypti larvae into pupae or adults marked with the damage on cuticles and necrosis on gastrointestinal epithel cells and respiratory tracts (9). Bruceine A isolated from the seed of Makasar fruit (B. javanica L. Merr) is also neurotoxic on larvae of $A$ e. aegypti through the prohibition on acetylcholinesterase enzyme and Voltage Gated Sodium Channel/ VGSC gene (10). Bruceine A and brusatol from Makasar fruit have the potential to be developed as natural larvicides and insecticides to vector disease control.

The purpose of this research was to find out the larvicidal activity of brusatol on $C x$. quinquefasciatus and its morphological changes/damages.

\section{Methods}

This research was conducted from May to September 2017. The extraction and isolation of brusatol from Makasar fruit was done in Pharmaceutical Biology Laboratory, Faculty of Pharmacy, Gadjah Mada University, Yogyakarta, Indonesia. The examination of larvicidal activity and morphological changes was conducted at the Parasitology Laboratory, Faculty of Medicine, Gadjah Mada University, Yogyakarta, Indonesia. Larvae $C x$. quinquefasciatus instar 3 up to the beginning of instar 4 were obtained and reared in the Parasitology Laboratory, Faculty of Medicine, Gadjah Mada University, Yogyakarta, Indonesia.

Extraction and isolation brusatol from B. javanica $L$. Merr

Brusatol extraction and isolation from the seeds of Makasar fruit was using Zhang et al. (7) method with slight modifications. Makasar fruit obtained from the traders of medicinal plants (Merapi Herbal Yogyakarta), identified in the Laboratory of Pharmaceutical Biology, Faculty of Pharmacy Gadjah Mada University, Yogyakarta, Indonesia. Chemicals and solvents used for the extraction and isolation of brusatol that are ethanol, methanol, chloroform, ethyl acetate and $\mathrm{H}_{2} \mathrm{O}$ were provided with the highest analytical quality obtained from Merck suppliers. Brucea javanica (L) Merr dried seeds (5 kg) made it into powder, shaken with EtOH-H2O (15 L), the solvent was evaporated in vacuum, and the extracts were combined and concentrated, followed by suspending in $\mathrm{H}_{2} \mathrm{O}$. The aqueous layer was further extracted with Petroleum ether, $\mathrm{CHCl}_{3}$, EtOAc and n-Butanol. The $\mathrm{CHCl}_{3}$ layer was evaporated under vacuum to afford extracts. This extract was re-suspended with $\mathrm{CHCl}_{3}$, and then chromatographed on a silica gel column (400 g, 200-300 mesh) by eluting successively with $\mathrm{CHCl}_{3}$ containing increasing amounts of $\mathrm{MeOH}(1: 0,50: 1$, $20: 1,10: 1,5: 1$ and $0: 1)$. The result in the organic layer was taken and evaporated to obtain a concentrate, and then made a solution of $\mathrm{MeOH}$ (100-250 mL) at a temperature of $60{ }^{\circ} \mathrm{C}$ and then stored at room temperature. $\mathrm{MeOH}$ solution above when it was allowed to stand at room temperature to form crystalline compounds that expected as brusatol. Further separation was by fil- 
tration. The rest of the filtrate / residue was separated by thin layer of chromatography (TLC). The filtrate / residue was evaporated. Further separation was performed if it was necessary.

\section{Larvicidal test of brusatol}

The larvicidal test was carried out using the bioassay method according to WHO (11) with slight modifications. The third instar larvae up to the beginning of instar 4 of $C x$. quinquefasciatus were acquired and allowed to develop at the Laboratory of Parasitology, Faculty of Medicine, University of Gadjah Mada. A preliminary test was conducted to determine the range of concentrations of brusatol that could be deadly to larvae of $C x$. quinquefasciatus instar 3 up to the beginning of instar 4. In further tests, temephos was used as a positive control at a concentration of $1 \mathrm{ppm}$, whereas the negative control consisted of $100 \mathrm{~mL}$ of distilled water only. The selection of temephos dosage (1 ppm) was based on lethal damage consideration used in the field. A total of 25 larvae of Cx. quinquefasciatus instar 3 up to the beginning of instar 4 were used in each treatment medium and control, replicated four times. After $24 \mathrm{~h}$, the dead larvae were counted. The temperature and $\mathrm{pH}$ of the media and humidity in the room were measured at the beginning and the end of the study.

\section{Larvicidal activity of brusatol}

Based on the isolation method from Zhang et al. (7), $150 \mathrm{mg}$ of brusatol isolated from $5 \mathrm{~kg}$ of Makasar fruit were obtained. The purity of the brusatol isolates analyzed by Thin Layer Chromatography (TLC) shows the presence of a purple single spot with an $\mathrm{Rf}$ value of 0.79 on $366 \mathrm{~nm}$ UV observation. The environment considered in this study was the $\mathrm{pH}$ of the media, media temperature, and humidity. These were measured at the beginning and end of the study as $\mathrm{pH} 7,25$ ${ }^{\circ} \mathrm{C}$, and $70 \%-71 \%$, respectively

\section{Morphology test}

Culex quinquefasciatus larvae in instar 3 up to the beginning of instar 4 were acquired and cultured at the Laboratory of Parasitology, Faculty of Medicine, University of Gadjah Mada. Morphological tests were conducted in accordance with the method reported by Sharma et al. (12) with slight modifications. Larvae were placed in a plastic cup containing $100 \mathrm{ml}$ of water and various concentrations of brusatol or $1 \mathrm{ppm}$ of the positive control temephos. Negative controls were treated with distilled water. Dead larvae were collected after $24 \mathrm{~h}$ of treatment for the examination of morphological changes under light microscopy. Larvae were scrutinized after mounting with Hoyer's medium and morphological changes in body segments including the head, setae, cuticle, abdomen, and anals gills were observed, photographed and compared with those of the controls.

\section{Data analysis}

The percentage of deaths of $C x$. quinquefasciatus larvae was expressed by Mean \pm Standard Error of Mean (SEM). The value of $\mathrm{LC}_{50}$ and $\mathrm{LC}_{90}$ brusatol was determined by using Probit regression analysis with SPSS version 24 (Chicago, IL, USA). To find out the difference between concentrations of brusatol in killing the larvae of Cx. quinquefasciatus, we used Kruskal Wallis test followed by Mann Whitney. Statistically significant differences were indicated at $P<0.05$. The data from observation on morphological changes were descriptively analyzed (13).

\section{Results}

The percentage of deaths of $C x$. quinquefasciatus larvae presented in Table 1 . The average values of Lethal Concentration $50\left(\mathrm{LC}_{50}\right)$ and Lethal Concentration 90 (LC $\mathrm{L}_{90}$ ) of brusatol against larvae of $C x$. quinquefasciatus were $0.010 \pm 0.122 \mathrm{ppm}$ and $0.654 \pm 0.081$ ppm respectively.

\section{Morphological changes of $C_{x}$. quinquefascia- tus}

The morphological observation of $C x$. quinquefasciatus larvae after complete brusatol and control treatment is presented in Table 2, Figs. 1 and 2. 
Bodies of control larvae (negative control) did not show any damages. Cx. quinquefasciatus larvae treated with various concentrations of brusatol and 1 ppm temephos exhibited damaged heads, cuticle, digestive tracts, breathing tract/tubes, siphons, and setae feathers.

Table 1: Percentage of the mortality of $C x$. quinquefasciatus larvae treated with brusatol, temephos of $1 \mathrm{ppm}$ and control after $24 \mathrm{~h}$ of observation

\begin{tabular}{lcccccccc}
\hline No. & $\begin{array}{c}\text { Concentration brusatol } \\
\text { (ppm) }\end{array}$ & $\begin{array}{c}\text { No. of } \\
\text { larva }\end{array}$ & \multicolumn{3}{c}{$\begin{array}{c}\text { Mortality of larvae on each } \\
\text { replicatoin }\end{array}$} & Mean & $\begin{array}{c}\text { Mortality } \pm \text { SEM } \\
(\%)\end{array}$ \\
\hline 1. & & & $\boldsymbol{I}$ & $\boldsymbol{I I}$ & $\boldsymbol{I I I}$ & $\boldsymbol{I V}$ & & \\
2. & 0.1 & 25 & 14 & 15 & 18 & 17 & 16.00 & $64 \pm 0.55^{*}$ \\
3. & 0.3 & 25 & 18 & 20 & 21 & 20 & 19.75 & $79 \pm 0.38^{*}$ \\
4. & 0.9 & 25 & 19 & 20 & 20 & 23 & 20.50 & $82 \pm 0.52^{*}$ \\
5. & 2.7 & 25 & 23 & 20 & 22 & 24 & 22.25 & $89 \pm 0.51^{*}$ \\
6. & 8.1 & 25 & 23 & 24 & 22 & 24 & 23.25 & $93 \pm 0.29^{*}$ \\
7. & 24.3 & 25 & 25 & 25 & 25 & 25 & 25.00 & $100 \pm 0.00^{*}$ \\
8. & Temephos 1ppm & 25 & 25 & 25 & 25 & 25 & 25.00 & $100 \pm 0.00$ \\
\hline
\end{tabular}

$* \mathrm{P}<0.05$ with Kruskal Wallis and Mann Whitney test; ppm: part per million; SEM: Standard Error of Mean
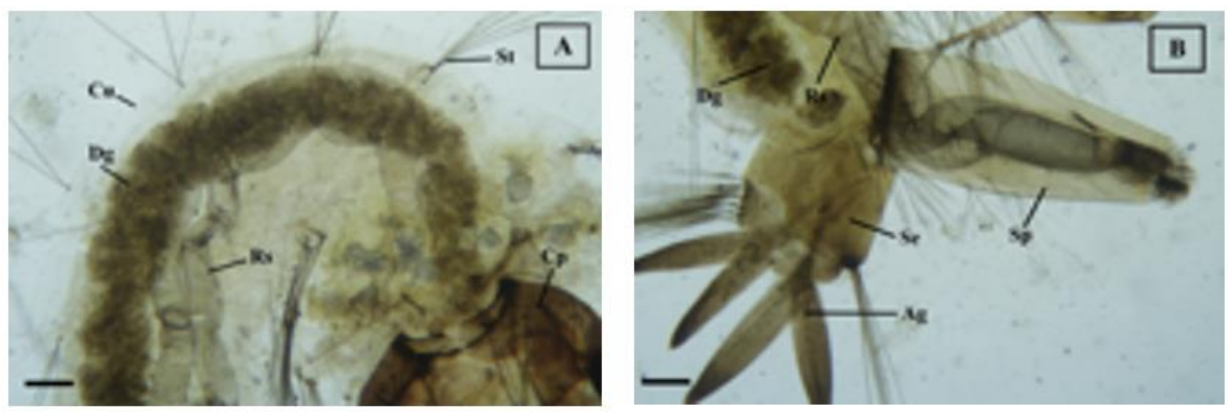

Fig. 1: Control of $C x$. quinquefasciatus larvae. Digestive and respiratory tract complete and well (A). Anterior body, (B). Posterior body (100x). Cp: caput, Dg: digestive tract, Rs: respiratory tract, Sp: siphon, Se: sandle, Cu: cuticle, Ag: anal gills, St: setae
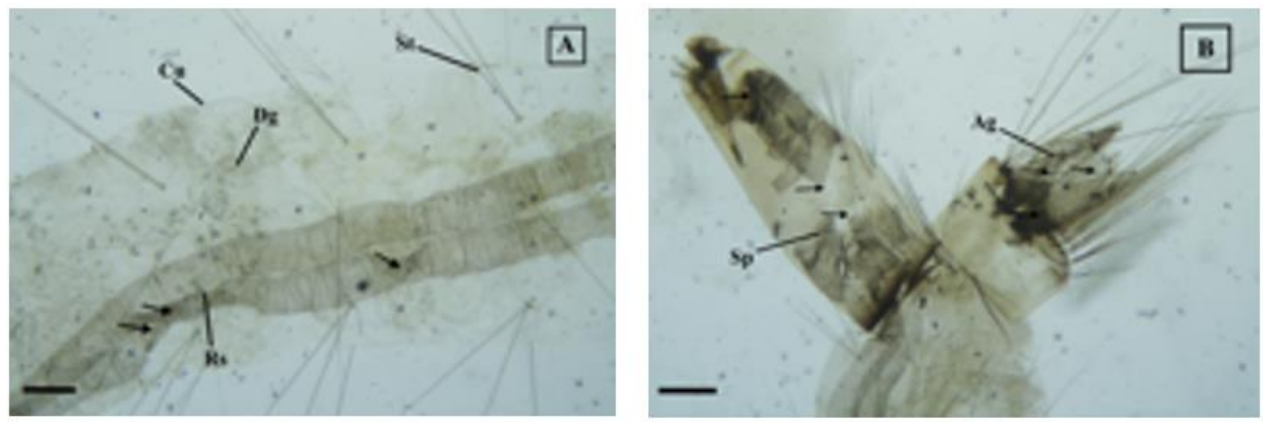

Fig. 2: Larvae of $C x$. quinquefasciatus treated with brusatol, (A). Brusatol concentration of $0.9 \mathrm{ppm}$. Respiratory tube folded, cuticle and feather setae detached in some parts, (B). Brusatol concentration of $0.1 \mathrm{ppm}$. Siphon and anal gills broken and detached (100x). Cp: caput, Dg: digestive tract, Rs: respiratory tract, Sp: siphon, Se: sandle, Cu: cuticle, Ag: anal gills, St: setae 
Table 2: Morphological changes of $C x$. quinquefasciatus larvae with control and brusatol treatment at various concentrations

\begin{tabular}{ll}
\hline Treatment & Morphological changes in $\boldsymbol{C} \boldsymbol{x}$. quinquefasciatus larvae \\
\hline $\begin{array}{l}\text { Brusatol } \\
24.3 \mathrm{ppm}\end{array}$ & $\begin{array}{l}\text { Damages on cuticle, digestive and respiratory tracts especially in the } \\
\text { siphon. }\end{array}$ \\
Brusatol & The body of the larvae and the head were blackened, the digestive tract \\
$8.1 \mathrm{ppm}$ & was damaged by a black spot, the respiratory tube was folded. \\
Brusatol & Blackened larval bodies, folds in respiratory tubes, damage to the ante- \\
$2.7 \mathrm{ppm}$ & rior part of the digestive tract and cuticle. \\
Brusatol & The digestive tract was damaged, the head was blackened. Respiratory \\
$0.9 \mathrm{ppm}$ & tube folded. There was no damage to the cuticle. \\
Brusatol & Many folds on the respiratory tube, damage to the siphon, the head was \\
$0.3 \mathrm{ppm}$ & blackened, no cuticle damage. \\
Brusatol & The respiratory tract/tube was folded, narrowed and damaged on the \\
$0.1 \mathrm{ppm}$ & inside of the siphon. \\
Temephos & The respiratory and digestive tracts are severe damage with some dark \\
$1 \mathrm{ppm}$ & spot. The siphon and anal gills were damaged/detached and blackened. \\
Control & Body condition intact and good, complete and good digestive system. \\
\hline
\end{tabular}

\section{Discussion}

Larvicidal activity of brusatol against $C_{X}$. quinquefasciatus

The $C x$. quinquefasciatus larvae used in this study were of instar 3 up to the beginning of instar 4. Larvae of instar 3 up to the beginning of instar 4 already have perfectly shaped organs, so death after treatment could not be attributed to the organs not being fully formed (14). The death of $C \times$. quinquefasciatus larvae was not caused by these environmental factors. The levels of $\mathrm{pH}$, temperature, and room humidity in the media were still within the optimal $\mathrm{pH}$ range (6.5-7), temperature $\left(25-27^{\circ} \mathrm{C}\right)$, and air humidity $(60 \%-$ $80 \%$ ) for the development of $C \times$. quinquefasciatus larvae in bioassay research (11).

The Pesticide Commission (15) states that a larvicide is said to be effective if the death/dying number of larvae reaches at least $90 \%$ within 24 hours. Based on 24 hours observation results showed that in the control group (without treatment) there was no death of $C x$. quinquefasciatus larvae, whereas at the lowest concentration of brusatol $(0.1 \mathrm{ppm})$ there were $64 \%$ mortality of the larvae.
From Table 1, it shows that the higher the concentration of brusatol the higher percentage of death of $C x$. quinquefasciatus larvae and statistically significant. Mortality of $C \times$. quinquefasciatus larvae $100 \%$ was found at the highest concentration of brusatol that was $24.3 \mathrm{ppm}$. This result is similar to previous research on Ae. aegypti larvae which proves that at the highest concentration of brusatol, it causes death of $100 \%$ larvae. At the lowest concentration of brusatol, it causes the death of Ae. aegypti larvae by $40 \%$ (8).

The difference in mortality of $C x$. quinquefasciatus larvae may be influenced by several factors: larvae instar and different sensitivity of each test larva species. The larval instar factor is related to the age of the test larvae. Although the larvae used are larvae instar 3 up to the beginning of instar 4 , but there are still variation in larval age. Differences in larval mortality are also influenced by differences in sensitivity of each test larva species. Although using larvae with the same instar and age, each larva has different levels of susceptibility. The interaction of toxic substances in the biological system is determined by the concentration of the test compound and the length of the test period (16). 
Deaths of $C x$. quinquefasciatus larvae of more than $90 \%$ were achieved at concentrations of brusatol $8.1 \mathrm{ppm}$ and $24.3 \mathrm{ppm}$. This proves that brusatol is effective as a natural larvacide in $C x$. quinquefasciatus larvae (15). Temephos at $1 \mathrm{ppm}$ concentration caused $100 \%$ mortality of $C x$. quinquefasciatus larvae, as well as at concentrations of $24.3 \mathrm{ppm}$ brusatol. The mortality of $C x$. quinquefasciatus larvae in this study was due to the toxic activity of the brusatol compounds acted as toxicants.

Brusatol toxicity on $C x$. quinquefasciatus larvae is expressed by Lethal Concentration 50 and 90 $\left(\mathrm{LC}_{50}, \mathrm{LC}_{90}\right)$, respectively $0.010 \pm 0.122$ and $0.654 \pm 0.081 \mathrm{ppm}$. Sutiningsih \& Nurjazuli (8), mentioned that brusatol toxicity $\left(\mathrm{LC}_{50}, \mathrm{LC}_{90}\right)$ in Ae. aegypti larvae respectively of $0.669 \pm 0.106$ and $8.331 \pm 0.060$. The smaller the Lethal Concentration value the more toxic a compound is (17). This means that brusatol is more toxic to $C x$. quinquefasciatus larvae than to Ae. aegypti larvae. The death of the mosquito larvae is caused by the inability of the larvae to detoxify the toxic compound that enters the body. The toxic compounds entering the larvae can cause four stages of response: excitation, convulsions, lysis (paralysis) and death (18). These toxic compounds cause disruption of the digestive system, respiratory system and nervous system in larvae (19-22). Brusatol enter the body of larvae through the mouth when the larvae were feeding. Brusatol is thought to decrease the activity of protease enzymes and food absorption and inhibit the taste receptors in the mouth area that will cause the larvae to fail to get a flavor stimulus, consequently the larvae are unable to recognize the surrounding food (23). Low feeding activity in the larvae causes the energy for the development of the larvae to be reduced so that the growth process is inhibited and eventually the larvae die. The bitter end of brusatol also causes food inhibition (antifeedant) in the test larvae. The bitter taste causes the larvae to not eat so the larvae starved and eventually die. Toxic compounds consumed by larvae will affect the amount and rate of eating so that resulted in decreased growth rate and survival ability. The tox- icity power comes from its toxic substances that contained in the biological compounds of Makassar fruits. The substance can be toxic through the absorption of the gastrointestinal tract or through the skin of the larvae (26). Brusatol isolated from B. javanica (L.) Merr. has insecticidal and antifeedant properties against instar 3 larvae of Spodoptera exigua (7). The active material contained in B. javanica (L.) Merr. extract has larvicidal effect against Crocidolomia pavonana (Lepidoptera: Crambidae) (24, 25). Extract of B. javanica (L.) Merr. can inhibit feeding, decrease the rate of growth and inhibit laying C. pavonana imago (25). Bruceine $\mathrm{A}$ isolated from the seeds of B. javanica (L.) Merr can inhibit the growth and development of $A$ e. aegypti larvae to become pupa/adult (9) .

\section{Morphological changes of $C_{x}$. quinquefascia- tus}

Observation of morphological changes is intended to determine the target of damaged organs/body parts of $C x$. quinquefasciatus larvae after treatment with brusatol. The morphological observation of $C x$. quinquefasciatus larvae after brusatol treatment showed differences compared with control (without treatment). From Fig. 1. it is seen that control of $C x$. quinquefasciatus larvae (without treatment) do not show any damage to their body parts. The condition of the larvae is intact and good. Digestive and respiratory tract complete and well.

Observation of morphology of $C x$. quinquefasciatus larvae after the treatment with lowest concentration of brusatol $(0.1 \mathrm{ppm})$ showed that the respiratory tube was folded, narrowed and damaged on the inside of the siphon. Anal gills were partially detached and damaged (Fig. 2B). At higher concentrations of brusatol (0.9 ppm) showed more severe damage to the respiratory tract with more folds and damaged at the end of the tube leading to siphon (Fig. 2A). The body of the larvae and the head were blackened, the digestive tract was damaged while the cuticle was not. The higher the concentration of brusatol, the morphological damage of $C x$. quinquefasciatus larvae was getting worse and wide- 
spread to cause damage to the digestive tract and cuticle. In addition, respiratory tubes, siphon and anal gills were having more severe damage.

Based on the results of this study, it proves that toxic substances in brusatol cause morphological damage in the body of $C \times$. quinquefasciatus larvae, such as head, cuticle, digestive tract, respiratory tract, siphon and anal gills. Brusatol is thought to enter the body of the larvae through the pores of the skin, the digestive tract / mouth and the respiratory tract/siphon (8). Toxic substances are relatively easier to penetrate the cuticle and extend into the body of the larvae (7). The number of toxic compounds that enter causes damage to skin cells. This toxic compound hydrolyzes the skin membrane by breaking down the skin protein (collagen) into several parts. The destruction of skin cell membranes resulted in the loss of impermeable membrane of the skin, so other toxic compounds are free to enter the body of the larvae (19). The large number of toxic compounds that enter causes the proteins in the skin membrane to be damaged, so the function of the skin as a body protector is disrupted (26).

Another way to insert brusatol into the body of $C x$. quinquefasciatus larvae is through the respiratory tract. Air enters through a siphon attached to the water surface (16). Brusatol toxic compounds is likely covering the surface of the medium thus blocking the siphon from obtaining oxygen from the surface of the medium $(26,27)$. Neural tissues of the larvae are very sensitive to the lack of oxygen causing the wither on the nerves and damage on the siphon and as a result the larvae have difficulty in breathing and eventually die (28).

Brusatol toxic compounds also cause electrolyte imbalance in the anal region causing larvae strongly bite anal gills and resulted in severe damage to the anal gills (Fig. 2B). This result is supported by previous studies on Ae. aegypti larvae after treatment with $9 \mathrm{ppm}$ of brusatol showed the presence of neurotoxic symptoms characterized by the aggressive circular motion of larvae biting the anal gills (8). Similar observations have also been reported by Warikoo and Kumar (29) on Ae. aegypti (L) larvae with Arge- mone mexicana root extract treatment concentration of $91.331 \mathrm{ppm}$ and 156.684 ppm for 24 hours, showed a structure deformity on anal gills. There was a structural deviation/ damage to anal papillary of early instar 4 of $A$ e. aegypti larvae after treatment with stem and leaf extract of Achyranthes aspera (12). The damages on anal papillae with shrinking cuticle has also been observed by Insun et al. (30) on the larvae $C x$. quinquefasciatus after treatment with ethanol extract of Kaempferia galanga. Anal gills damage causes impaired function in osmotic and ionic regulation (20). This disorder of regulatory function is expected to be the cause of death of larvae $C \times$. quinquefasciatus. Brusatol can induce apoptosis in the insect cell line IOZCAS- Spec-II and Sf21 characterized by DNA fragmentation, caspase- 3 activation and release of cytochrome-c enzyme from mitochondria (7).

Damage to the gastrointestinal tract and siphon in $C x$. quinquefasciatus larvae began to be seen in treatment with brusatol at the lowest concentration (0.1 ppm). Digestive tract of $C \times$. quinquefasciatus larvae began to show the damage after brusatol treatment concentration of 0.9 ppm (Table 2). The results of this study were not different from previous studies, proved that bruceine $A$ at $0.2 \mathrm{ppm}$ concentration causes damage to the digestive and respiratory tracts of Ae. aegypti larvae (8). Distortion in the middle of the gastrointestinal tract (midgut) with a loss of pigmentation and a partial or total damage to cells in the larvae of $A$ e. aegypti after treatment with stem and leaves extract of $A$. aspera (12). Microscopic observation by using a light/electron microscope showing at observations of 6,12, 24 and 48 hours, there was an increase of damage in epithelial midgut after treatment with $A$. aspera. Similar observations in midgut were also reported by Chaithong et al. (21) in Ae. aegypti larvae after treatment with pepper extract. Damage to the gastrointestinal tract and respiratory caused the metabolism and respiration of the larvae were disturbed so that the larvae became dead.

The results of this research suggest that brusatol can be categorized as abdominal and respiratori- 
cal toxic because it kills $C x$. quinquefasciatus larvae with action target on gastrointestinal and respiratory tract. These results also support the potential of natural, plant-based larvicides for environmentally sensitive control of disease vectors such as Cx. quinquefasciatus.

This study will help the researchers to discover the critical areas of target site of brusatol, which has not been explored yet. Future studies should examine how brusatol affects growth hormone production as well as molting and metamorphosis also detailed description of histological damage by microscopic observation using transmission electron microscopy and fluorescence imaging of cell viability markers via confocal microscopy.

\section{Conclusion}

Brusatol isolated from B. javanica (L.) Merr has a natural larvacidal activity in $C x$. quinquefasciatus larvae that can be used to control the population of $C x$. quinquefasciatus larvae as disease vectors. The larvicidal activity of brusatol is indicated by morphological damage to the body parts of $C x$. quinquefasciatus larvae, such as in: head, cuticle, respiratory tract, siphon, digestive tract and anal gills.

\section{Ethical considerations}

Ethical issues (Including plagiarism, informed consent, misconduct, data fabrication and/or falsification, double publication and/or submission, redundancy, etc.) have been completely observed by the authors.

\section{Acknowledgments}

The authors would like to thank the Rector of University of Diponegoro who has funded this study through APBN DPA SUKPA LPPM University of Diponegoro funding No. 275028/UN7.5.1/PG/ 2017, dated March 23, 2017.

\section{Conflict of interest}

The authors declare that there is no conflict of interests.

\section{References}

1. Jansen CC, Beebe N (2010). The dengue vector of Aedes aegypti: What comes next. Microbes Infect, 12 (4):272-9.

2. Isman MB (2006). Botanical insecticides, deterrents, and repellents in modern agriculture and an increasingly regulated world. Annu Rev Entomol, 51:45-66.

3. Isman MB (2008). Botanical insecticides: for richer, for poorer. Pest Manag Sci, 64 (1):8-11.

4. Takeya K, Kim I, Hitotsuyanagi Y, Hasuda T (2006). Antitumour quassinoid from Brucea javanica and SAR of Brusatol. Planta Med, 72:20 4.

5. Govindachari T, Krishna KGN, Gopalakrishnan G, Suresh G, Wesley S, Seelatha T (2001). Insect antifeedant and growth regulating activities of quassinoids from Samadera indica. Fitoterapia, 72:568-71.

6. Chen YY, Pan Q, Li D, Liu J, Wen Y, Huang Y, Lu F (2011). New pregnane glycosides from Brucea javanica and their antifeedant activity. Chem Biodivers, 8:460-6.

7. Zhang L, Feng X, Ma D, Yang J, Jiang H, Zhang $\mathrm{Y}, \mathrm{He} \mathrm{W}$ (2013). Brusatol isolated from Brucea javanica (L) Merr induces apoptotic death of insect cell lines. Pestic Biochem Physiol, 107 (1):18-24.

8. Sutiningsih D, Nurjazuli (2017). Effect of brusatol biolarvicide administration on behavioral response of Aedes aegypti and its toxicity on Vero cells. J Biol Sci, 17:127-35.

9. Sutiningsih D, Mustofa, Satoto TBT, Martono E (2017). Inhibitory effects of bruceine A biolarvicide on growth and development of Aedes aegypti larvae. J Entomol, 14:104-11.

10. Sutiningsih D, Mustofa, Satoto TBT, Martono E (2017). Neurotoxic mechanism of bruceine A biolarvicide against Aedes aegypti Linnaeus larvae. Res J Med Plants, 11:77-85.

11. WHO (2005). Guidelines for laboratory and field testing of mosquito larvicides. World Health Organization. Geneva.

12. Sharma A, Kumar S, Tripathi P (2015). Impact of Achyranthes aspera leaf and stem extracts on the survival, morphology and behaviour of an 
Indian strain of dengue vector, Aedes aegypti $\mathrm{L}$ (Diptera: Culicidae). JMR, 5 (7):1-9.

13. Dahlan M (2014). Statistic for medicine and health. 6 th ed. Indonesian Epidemiology. Jakarta.

14. Farnesi LC, Brito JM, Linss JG, Pelajo-Machado M, Valle D, Rezende GL (2012). Physiological and morphological aspect of Aedes aegypti developing larvae: Effects of the chitin synthesis inhibitor novaluron. PloS ONE, 7 (1): e30363.

15. Pesticide Commission (1995). Standard method of pesticide effication test. Department of Agriculture. Jakarta.

16. Matsumura F (1985). Toxicology of insecticides. $2^{\text {nd }}$ ed. Plenum Press. New York.

17. Wahyuni D (2015). New bioinsecticide granules toxin from extract of papaya (Carica papaya) seed and leaf modified against Aedes aegypti larvae. Procedia Environ Sci, 23:323-8.

18. Sanjaya $\mathrm{Y}$, Safaria $\mathrm{T}$ (2006). Toxicity poison from spider Nepbila sp. to Aedes aegypti L. larvae. J Biodiversitas, 7:191-4.

19. Choochote W, Tuetun B, Kanjanapothi D et al (2004). Potential of crude seed extract of celery, Apium graveolens L., against the mosquito Aedes aegypti (L.) (Diptera: Culicidae). J Vector Ecol, 29 (2):340-6.

20. Dharmagadda VS, Naik SN, Mittal PK, Vasudevan P (2005). Larvicidal activity of Tagetes patula essential oil against three mosquito species. Bioresour Tecbnol, 96 (11):1235-40.

21. Chaithong U, Choochote W, Kamsuk K et al (2006). Larvicidal effect of pepper plants on Aedes aegypti (L.) (Diptera: Culicidae). J Vector Ecol, 31 (1):138-44.

22. Kumar S, Warikoo R, Wahab N (2010). Larvicidal potential of ethanolic extracts of dried fruits of three species of peppercorns against different instars of an Indian strain of dengue fever mosquito, Aedes aegypti L. (Diptera: Culicidae). Parasitol Res, 107 (4):901-7.

23. Tjokropranoto R, Evacuasiany E, Saputro N (2010). Efectiveness of Beluntas infusion (Plucea indica L.) as larvicide againts Aedes sp. Med Planta, 1 (2):75-80.

24. Syahputra E (2008). Bioactivity of Brucea javanica fruit as nabatical insecticide for agricultural insect pest. Bul Littro, 19 (1):56-67.

25. Lina E, Dadang, Manuwoto S, Syahbirin G, Prijono D (2013). Synergistic action of mixed extract Brucea javanica (Simaroubaceae), Piper aduncum (Piperaceae), and Tephrosia vogelli (Leguminosae) against cabbage head caterpillar, Crocidolomia pavonana. J Biopestic, 6 (1):77-83.

26. Lu F, Kacew S (2002). Lu's basic toxicology: Fundamentals, taryets organ and risk assesment. $4^{\text {th }} \mathrm{ed}$. Taylor \& Francis. New York.

27. Wulandari S, Arnetis, Rahayu S (2012). Potential of sap Papaya fruit (Carica papaya L) against mortality of Aedes albopictus mosquitoes larvae. Biogenesis, 9 (1):69-75.

28. Kringer R (2010). Handbook of pesticide toxicology. Academic Press. California.

29. Warikoo R, Kumar S (2013). Impact of Argemone mexicana extracts on the cidal, morphological, and behavioural response of dengue vector, Aedes aegypti L. (Diptera: Culicidae). Parasitol Res, 112 (10):3477-84.

30. Insun D, Choochote W, Jitpakdi A, Chaithong U, Tippawangkosol P, Pitasawat B (1999). Possible site of action of Kaempferia galanga in killing Culex quinquefasciatus larvae. Southeast Asian J Trop Med Public Health, 30 (1):195-9. 